\title{
Estudo de propriedades dinâmico-mecânicas de um compósito aeronáutico de CFRP com fios de ligas com memória de forma embebidos
}

\section{Dynamic-mechanical properties study of an aeronautical CFRP composite with embedded shape memory alloy wires}

\author{
Zoroastro Torres Vilar ${ }^{1 *}$, Carlos José de Araujo² e Abdias Gomes dos Santos ${ }^{2}$ \\ ${ }^{1}$ Universidade Federal Rural do Semi-Árido - UFERSA, Mossoró, RN, Brasil \\ ${ }^{2}$ Universidade Federal de Campina Grande - UFCG, Campina Grande, PB, Brasil \\ *zoroastro@ufersa.edu.br
}

\begin{abstract}
Resumo
Este trabalho trata-se de um estudo experimental do comportamento de propriedades termomecânicas de um compósito ativo, obtido através da introdução de diferentes frações volumétricas de fios de uma liga NiTi com efeito de memoria de forma em uma matriz polimérica reforçada com fibras de carbono (CFRP). Para tal estudo, inicialmente fez-se uma análise do módulo elástico de um pré preg de CFRP aeronáutico com diferentes posições de ângulo de fibras de carbono, a fim de determinar a matriz mais adequada para a introdução de fios de NiTi para obter compósito ativo. Verificou-se que a matriz com fibras alinhadas a $15^{\circ}$ apresenta-se como uma boa opção para a obtenção de compósitos ativos, sendo utilizada para a fabricação de amostras de CFRP/NiTi. As amostras tiveram seu potencial de ativação avaliado através da análise da variação do módulo de elasticidade com o aumento da temperatura usando a técnica de Análise Dinâmico-Mecânica (DMA). Através dos resultados obtidos, verificou-se a capacidade de ativação dos compósitos CFRP/NiTi, os quais apresentam variação positiva de módulo de elasticidade ao serem aquecidos acima da temperatura de transformação dos fios NiTi.
\end{abstract}

Palavras-chave: compósitos ativos, CFRP, ligas com efeito de memoria de forma, análise dinâmico-mecânica.

\begin{abstract}
This paper is in an experimental study of the thermomechanical properties behavior of an active composite obtained by introducing different volumetric fractions of NiTi shape memory alloy wires into a reinforced carbon fiber polymer (CFRP) matrix. For this study, an initial analysis of an aeronautic CFRP pre-preg with different angle positions of carbon fibers was performed verifying the elastic modulus in order to determinate the more adequate matrix for introducing NiTi SMA wires to obtain active composites. It was found that the CFRP aligned with the carbon fibers at $15^{\circ}$ presents with a good choice for obtaining active composite, being used to manufacture CFRP/NiTi samples. The samples had their activation potential confirmed by the change in elastic modulus with increasing temperature using the Dynamic Mechanical Analysis (DMA) technique. By obtained results, we verified the activation ability of the CFRP/NiTi composite, which show positive variation of elastic modulus when heated above transformation temperatures of the NiTi wires.
\end{abstract}

Keywords: active composites, CFRP, shape memory alloys, dynamic-mechanical analysis.

\section{Introdução}

Compósitos ativos ou inteligentes (termo também utilizado) são estruturas capazes de detectar mudanças no funcionamento e/ou condições ambientais e promover adaptações através da ativação de atuadores, procurando manter um comportamento satisfatório, previamente estabelecido $^{[1]}$. São sistemas que contém inerente em sua estrutura as potencialidades de atuar, detectar e controlar, através da combinação de vários materiais ${ }^{[2]}$, oferecendo propriedades que variam de acordo com a solicitação do meio que os envolve, ajustando seu comportamento e otimizando o desempenho da estrutura.
Dentre os materiais funcionais empregados no desenvolvimento de estruturas ativas, destacam-se as ligas com efeito de memória de forma (LMF), por suas propriedades termomecânicas de geração de força e/ou deformação em função da temperatura, além da capacidade de "sentir" e atuar. Essas LMF, internacionalmente conhecidas por Shape Memory Alloys (SMA), são geralmente empregadas na forma de fios que possuem qualidade elevada como atuador termomecânico mesmo com diâmetros abaixo de $0,2 \mathrm{~mm}$. Esse fato permite a sua integração direta em compósitos 
reforçados com fibra em matriz polimérica, sem comprometer a integridade estrutural do material ${ }^{[3]}$.

A incorporação de LMF na forma de fios finos aos compósitos poliméricos reforçados com fibras de carbono (CFRP - Carbon Fiber Reinforced Polymer) já foi objeto de alguns poucos estudos e pode resultar em estruturas ativas, capazes de provocar alterações em propriedades importantes como rigidez, amortecimento e frequência natural da estrutura, além de dota-las da capacidade de auto adaptação às condições existentes no meio, ou exibirem propriedades variáveis com as necessidades ${ }^{[3-5]}$.

Neste contexto, para esse trabalho foram fabricadas pequenas placas de compósitos ativos, através da incorporação de fios de LMF NiTi em folhas pré-impregnadas de CFRP, com as fibras de carbono posicionadas em ângulo em relação aos fios $\operatorname{NiTi}\left(15^{\circ} / 0^{\circ} / 15^{\circ}\right)$. O potencial de ativação dos compósitos CFRP/NiTi obtidos foi investigado usando caracterização termomecânica através da aplicação da técnica de Análise Dinâmico-Mecânica (DMA).

\section{Procedimento Experimental}

Os compósitos desenvolvidos neste estudo consistem basicamente de quatro camadas de pré-impregnados de fibras de carbono unidirecionais, fornecidos pela empresa Hexcel Composites (UK) contendo diferentes frações volumétricas de fios LMF NiTi, distribuídos uniformemente ao longo do plano neutro das amostras. O pré-preg utilizado (HexPly ${ }^{\circledR}$ 8552) é um compósito desenvolvido para aplicações aeroespaciais e consiste de aproximadamente 57,5\% em volume de fibras de carbono imersas em uma resina epóxi de alta resistência ${ }^{[6]}$.

Pelo fato desse compósito apresentar módulo de elasticidade superior as dos fios NiTi a serem introduzidos para a obtenção de compósitos ativos, inicialmente realizou-se uma análise da influência do ângulo das fibras de carbono em relação à direção dos fios NiTi para que o compósito obtido apresentasse módulo de elasticidade com valor próximo ao dos fios NiTi no estado martensítico (não ativado). Para esse estudo, obtiveram-se amostras de CFRP (sem fios NiTi) em diferentes ângulos (de 0 a $45^{\circ}$ com incrementos de $5^{\circ}$ ) em relação ao eixo longitudinal, através do corte de uma única placa de CFRP nas angulações desejadas.

Após a obtenção de amostras de CFRP em diferentes ângulos, foram realizados testes em DMA e através de uma análise teórica usando a regra das misturas ${ }^{[7]}$, selecionou-se a amostra com angulação de fibras que apresentou propriedades proximas as dos fios NiTi. Assim, posteriormente foram produzidos compósitos de CFRP/NiTi com ângulo das fibras de carbono em relação aos fios NiTi de $15^{\circ}$ e com diferentes frações volumétricas de fios NiTi. Tais amostras foram fabricadas com fios NiTi da liga M (diâmetro de 0,29 mm) e liga $\mathrm{H}$ (diâmetro de $0,30 \mathrm{~mm}$ ) fornecidos pela empresa alemã Memory Metalle ${ }^{[8]}$. Essas amostras foram denominadas de CFRP/NiTi-M e CFRP/NiTi-H, respectivamente.

Os fios foram embebidos na matriz de CFRP (com angulação selecionada através dos testes de DMA) em duas condições: tratados termicamente e treinados após tratamento térmico. $\mathrm{O}$ treinamento leva a uma estabilização das propriedades termomecânicas dos fios NiTi e consistiu de uma sequência de 1000 ciclos de aquecimento por efeito Joule e resfriamento ao ambiente (em torno de $30^{\circ} \mathrm{C}$ ), sob carga constante aplicada ao fio $\mathrm{NiTi}$, conforme descrito em trabalho anterior de Araújo et al. ${ }^{[5]}$. Por meio da Figura 1 mostra-se de forma esquemática a configuração das amostras de viga retangular CFRP/NiTi produzidas para este estudo. Os fios NiTi foram alinhados no sentido longitudinal da amostra e o pre-preg foi cortado no formato retangular com as fibras na angulação selecionada, sendo posicionadas camadas de CFRP acima e abaixo dos fios. As dimensões de $5,5 \times 25 \mathrm{~mm}$ foram selecionadas considerando a utilização do equipamento de DMA modelo Q800 da TA Instruments, com as amostras testadas em modo de viga simplesmente engastada (single cantilever). Para a fabricação das amostras de CFRP seguiu-se o ciclo de cura proposto pela empresa Hexcel Composites (UK), que consiste de prensagem uniaxial a quente por quatro horas, a uma temperatura de $120^{\circ} \mathrm{C}$ e uma pressão de $0,32 \mathrm{MPa}$, seguido de resfriamento ao ar. O processo foi realizado em uma máquina universal de ensaios (Instron, model 5582) com câmara de aquecimento, usando um molde metálico projetado especificamente para essa finalidade, que alem de alinhar os fios NiTi, também os mantem presos durante o processo de cura ${ }^{[7]}$.

Na Figura 2 mostra-se de forma esquemática o dispositivo desenvolvido para alinhar e prender os fios NiTi durante o processo de cura. Os fios foram inicialmente presos nas distancias desejadas e em seguida aplicou-se uma pré-carga mínima (rosqueando-se as porcas das hastes longitudinais), necessária apenas para garantir o alinhamento dos fios NiTi.

Os fios NiTi utilizados foram submetidos a tratamento térmico com aquecimento a uma temperatura de $450{ }^{\circ} \mathrm{C}$ durante 30 minutos, com posterior resfriamento ao ar e decapagem em solução química $\left(3 \% \mathrm{HF}+15 \% \mathrm{HNO}_{3}\right.$ $+82 \% \mathrm{H}_{2} \mathrm{O}$ in vol. $\%$ por 10 minutos), seguindo metodologia proposta por Xu et al. ${ }^{[8]}$, para remoção de alguma camada de óxido eventualmente depositada na superficie dos fios NiTi.

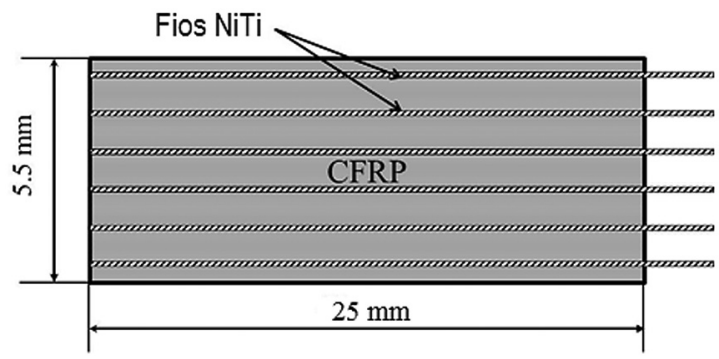

Figura 1. Representação esquemática das amostras de compósito ativo CFRP/NiTi.

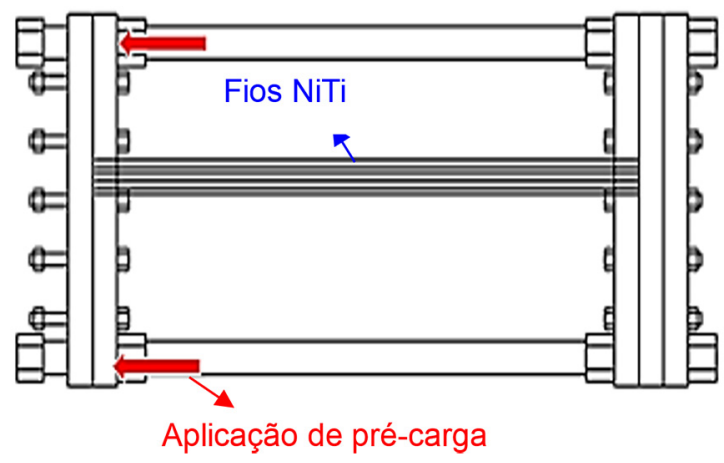

Figura 2. Ilustração esquemática do dispositivo desenvolvido para alinhar os fios NiTi. 
Os ios NiTi, o pré-preg de CFRP e os compósitos CFRP/NiTi obtidos foram caracterizados por análises térmicas em DSC e DMA, visando determinar, respectivamente, as temperaturas de transformação de fase dos fios NiTi e o comportamento do módulo de elasticidade (E) em função da temperatura do CFRP e também dos fios NiTi.

Para os ensaios em DMA as amostras de CFRP puro e CFRP/NiTi foram testadas em flexão no modo single cantilever e em tração utilizando-se uma freqüência de $2.5 \mathrm{~Hz}$ com uma amplitude de $15 \mu \mathrm{m}$ para o modo de flexão e $0,015 \%$ do comprimento útil da amostra para o modo de tração. As amostras foram aquecidas da temperatura ambiente $\left(\sim 30^{\circ} \mathrm{C}\right)$ até $120^{\circ} \mathrm{C}$ com uma taxa de aquecimento de $2{ }^{\circ} \mathrm{C} / \mathrm{min}$. Para os ensaios dos fios LMF NiTi em DSC utilizou-se essa mesma taxa de aquecimento $\left(2{ }^{\circ} \mathrm{C} / \mathrm{min}\right.$.), sem repetições em ambos os testes. Foi realizada também uma análise microscópica nas duas amostras de CFRP/NiTi produzidas com os diferentes fios LMF. Os ensaios de DMA e DSC foram realizados em equipamentos da TA Instruments, modelos DMA Q800 e DSC Q20, enquanto a análise microscópica foi realizada usando um microscópio ótico Olympus, modelo BX51.

\section{Resultados e Discussões}

Inicialmente, antes da fabricação dos compósitos Híbridos com Ligas com Efeito de Memória de Forma - CHLMF, realizou-se uma caracterização prévia dos fios de LMF NiTi e do compósito CFRP puro para determinar as suas propriedades termomecânicas. Através da análise em DSC determinou-se as temperaturas de transformação de fase
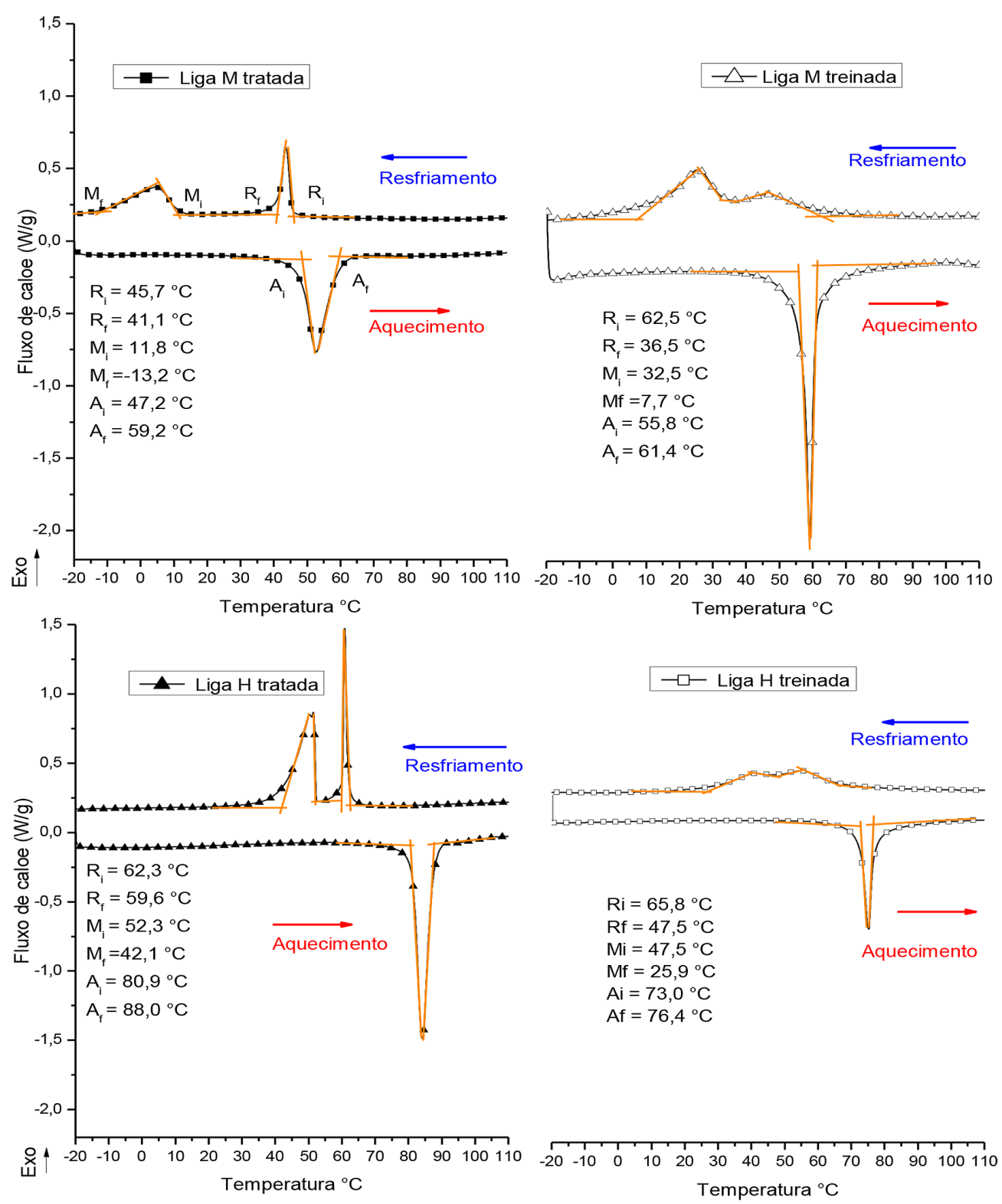

Figura 3. Variação do fluxo de calor em função da temperatura para os fios LMF NiTi. 
dos fios NiTi, de ambas as LMF NiTi (M e H). Na Figura 3 mostra-se os resultados obtidos através dessa técnica, com a variação do fluxo de calor em função da temperatura para as quatro amostras de fios NiTi utilizadas. Aplicando o método das tangentes aos picos de DSC, conforme indica a norma ASTM F2082-06 $6^{[9]}$, determinou-se as temperaturas de transformação de fase para os dois fios NiTi nos dois estados mencionados anteriormente. Na primeira curva de DSC, para o fio NiTi da liga M tratada, é mostrada a determinação de todas as temperaturas de transformação possíveis de ocorrer nesse material ${ }^{[10]}$ : $\mathrm{R}_{\mathrm{i}}$ e $\mathrm{R}_{\mathrm{f}}$, referentes ao início e final da transformação da fase austenita em martensita romboédrica (fase $\mathrm{R}$ ), $\mathrm{M}_{\mathrm{e}}$ e $\mathrm{M}_{\mathrm{f}}$, referentes ao início e final da transformação da fase martensita romboédrica em martensita monoclínica, ambas durante resfriamento, além das temperaturas $\mathrm{A}_{\mathrm{i}}$ e $\mathrm{A}_{\mathrm{f}}$, referentes ao início e final da transformação de fase reversa, da martensita monoclínica em austenita cúbica, durante o aquecimento.

Comparando-se os dois tipos de fios (Ligas $\mathrm{M}$ e H) através dos resultados apresentados na Figura 3 observa-se que as temperaturas de transformação de fase são maiores, e situadas na faixa de $30^{\circ} \mathrm{Ca} 100^{\circ} \mathrm{C}$, para os fios NiTi da liga $\mathrm{H}$ em comparação com os fios da liga M. Isso ocorre pelo fato dos fios NiTi da liga M apresentarem maior quantidade de níquel com relação aos da liga $\mathrm{H}^{[8]}$, já que o aumento da quantidade níquel diminui as temperaturas de transformação de fase pela formação de precipitados ricos em $\mathrm{Ni}^{[10]}$.

Verifica-se também na Figura 3 que o processo de treinamento tem uma discreta influencia nas temperaturas de inicio de ativação dos fios $\operatorname{NiTi}\left(\mathrm{A}_{\mathrm{i}}\right)$, levando a um leve aumento (liga M) ou diminuição (liga H) da ordem de $7{ }^{\circ} \mathrm{C}$.

Nas Figuras 4 e 5 são mostradas as variações do módulo de elasticidade (E) em função da temperatura durante aquecimento, para os fios NiTi (ligas M e H) e do compósito CFRP puro com as fibras de carbono alinhadas a $\theta=0^{\circ}$, ensaiados no modo de tração. Uma comparação entre os resultados destas duas figuras permite verificar o comportamento oposto dos dois constituintes dos compósitos ativos CFRP/NiTi, ou seja, enquanto os fios NiTi aumentam de rigidez durante o aquecimento entre $60^{\circ} \mathrm{C}$ e $140{ }^{\circ} \mathrm{C}$, a matriz de CFRP apresenta uma redução substancial de módulo de elasticidade logo após a temperatura de transição vítrea $\left(T_{G} \sim 125^{\circ} \mathrm{C}\right)$.

Através dos resultados obtidos no ensaio de DMA, constata-se que ambas os fios LMF apresentam aumento substancial de rigidez quando aquecidos até uma temperatura suficientemente alta para promover a transformação de fase. $\mathrm{O}$ aumento observado de módulo de elasticidade dos fios NiTi indica um grande potencial para obtenção de estruturas ativas. Os resultados apresentados na Figura 4 revelam ainda que os fios NiTi apenas tratados termicamente apresentaram maiores aumentos de módulo de elasticidade do que os fios treinados. Esses aumentos foram ainda maiores para os fios NiTi da liga M. O módulo de elasticidade, para o fio NiTi da liga $\mathrm{H}$ cresce de $21 \mathrm{GPa}$ para aproximadamente $45 \mathrm{GPa}$ quando totalmente austenítico e de $22 \mathrm{GPa}$ para $52 \mathrm{GPa}$ no caso dos fios NiTi da liga M. Para ambos os fios NiTi obteve-se um aumento de modulo superior a $100 \%$. Para os fios treinados de ambas as ligas, também se observaram aumentos de módulo de elasticidade, entretanto inferiores aos dos fios NiTi apenas tratados. Esse comportamento pode ser atribuído ao efeito da introdução de defeitos internos, como martensita residual, aumento da densidade discordâncias, gerados durante o processo de treinamento (ciclagem térmica sob carga de $200 \mathrm{MPa}$ ) dos fios $\mathrm{NiTi}^{[10]}$.

Através da aplicação do método das tangentes as curvas $E(T)$ da Figura 4, determinaram-se as temperaturas em que os fios NiTi iniciam e terminam a transformação de fase reversa, de martensita em austenita $\left(\mathrm{A}_{\mathrm{i}}\right.$ e $\left.\mathrm{A}_{\mathrm{f}}\right)$. Para os fios NiTi estudados observa-se que a liga $\mathrm{M}$ (tratada e treinada) apresenta temperaturas de transformação de fase inferiores aos fios da liga $\mathrm{H}$, conforme previsto pela empresa fornecedora Memory-Metalle ${ }^{[11]}$. Conforme já discutido, isso ocorre devido a maior quantidade de níquel fios NiTi da liga M.

Analisando os parâmetros relativos à temperatura de mudança de fase e aumento de rigidez (módulo de elasticidade) dos fios NiTi utilizados nesse estudo, aqueles da liga M no estado tratado apresentam-se como melhor opção para obter estruturas ativas. Além de apresentarem maiores valores de aumento de módulo em uma faixa de temperatura pequena, esse aumento acontece em temperaturas relativamente baixas, situadas entre $70^{\circ} \mathrm{C} \mathrm{e} 100{ }^{\circ} \mathrm{C}$ facilitando a sua incorporação em matrizes poliméricas por não haver a necessidade de

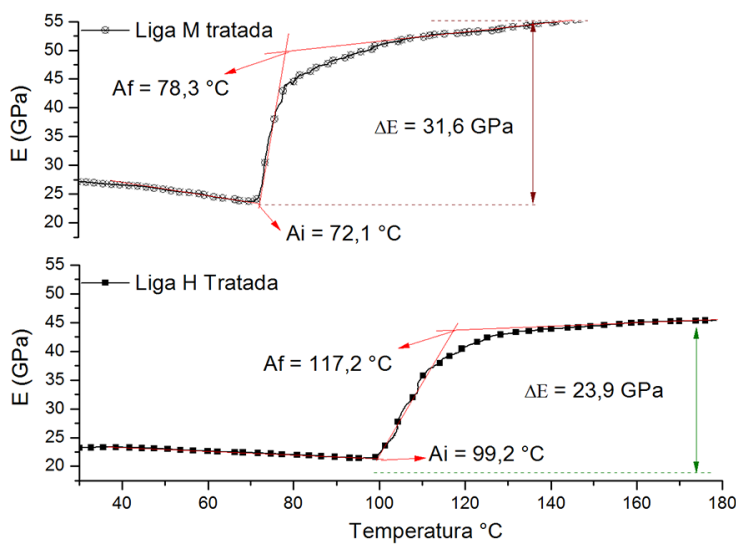

(a)

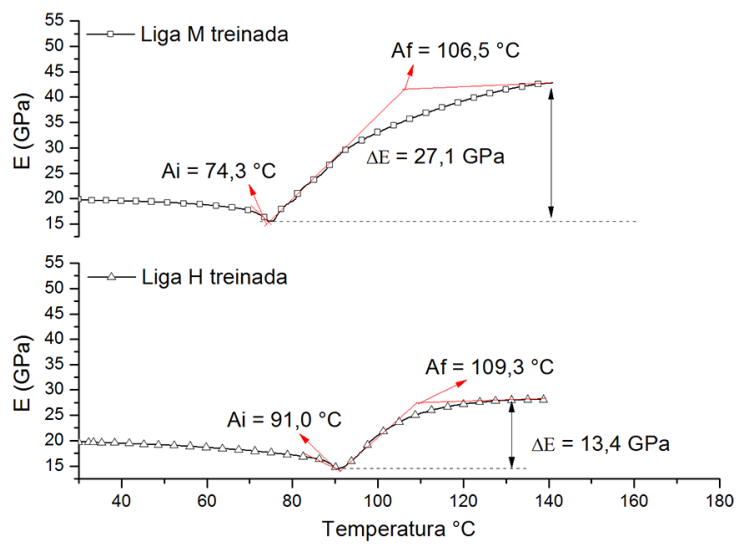

(b)

Figura 4. Variação do módulo de elasticidade em função da temperatura durante o aquecimento dos fios NiTi tratados (a) e treinados (b). 
submeter à matriz a altas temperaturas (muito superiores $\mathrm{a} \mathrm{T}_{\mathrm{g}}$ ) que possam causar a sua instabilidade térmica.

Apesar da análise em DMA constituir a principal técnica a ser empregada nesse estudo, enfrentou-se algumas limitações para a sua utilização, devido a necessidade de usar nitrogênio líquido para o resfriamento das amostras até uma temperatura suficientemente baixa para promover a transformação direta dos fios NiTi para a fase martensítica. Dessa forma, não foi possível realizar a caracterização durante o resfriamento.

Os valores das temperaturas de transformação de fase reversa obtidas para os fios NiTi em DMA (variação de módulo de elasticidade) e DSC durante o aquecimento, assim como a variação do módulo elástico $(\Delta \mathrm{E})$ são resumidos na Tabela 1.

Comparando os valores de temperaturas de transformação obtidos em DMA durante o aquecimento (Tabela 1) com os obtidos em DSC observa-se a existência de diferenças entre as temperaturas medidas. Esse fato é associado a fenômenos de alinhamento da martensita por deformação, já que a técnica de DMA aplica deformações durante o ensaio, assim como por transferência de calor que amostras metálicas sofrem quando ensaiadas em DMA, de forma que a temperatura da amostra é inferior à temperatura medida na câmara de aquecimento do equipamento. Isso ocorre devido à condução de calor da amostra metálica para a garra do DMA, que por possuir uma massa relativamente maior, se comparada com a amostra, acaba demorando mais a aquecer e dessa forma sua temperatura real é inferior à temperatura medida pelo equipamento. Dessa forma, como a transferência de calor por condução da amostra para a garra é elevada, o fio NiTi também estará a uma temperatura inferior a medida pelo

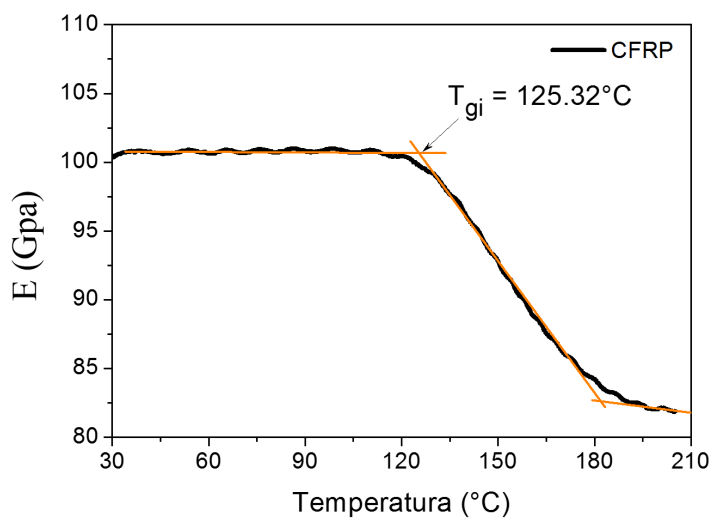

Figura 5. Variação do módulo de elasticidade em função da temperatura para o compósito de CFRP puro com fibras alinhadas a $0^{\circ}$. equipamento, havendo assim diferença entre essas duas temperaturas (DSC e DMA) ${ }^{[12]}$.

Analisando as temperaturas de transformação de fase direta (austenita para martensita) durante o resfriamento em DSC (Figura 3), observa-se que os fios NiTi da liga $\mathrm{H}$ apresentam-se totalmente martensíticos na temperatura ambiente, enquanto que o fio NiTi proveniente da liga $\mathrm{M}$ encontra-se em uma fase intermediaria, correspondente a uma martensita romboédrica conhecida por fase $\mathrm{R}^{[10]}$. Diante disso, como as amostras de compósitos ativos (CRFP/NiTi) produzidas são testadas em DMA da temperatura ambiente (aproximadamente $30^{\circ} \mathrm{C}$ ) até uma temperatura que permita a total transformação de fase dos fios NiTi dentro da matriz CFRP, os fios da liga $\mathrm{M}$ não terão sua transformação martensítica completamente atingida e sua total eficiência pode não ser alcançada.

Na Figura 5 mostram-se resultados da caracterização das propriedades dinâmico-mecânicas do compósito CFRP puro com as fibras de carbono alinhadas $\left(\theta=0^{\circ}\right)$, a ser usado como matriz para a incorporação dos fios NiTi. Notam-se as suas excelentes propriedades mecânicas assim como a estabilidade térmica dessas propriedades até temperaturas da ordem de $120^{\circ} \mathrm{C}$, suficiente para ativação dos fios NiTi. O módulo de elasticidade inicial do CFRP está em torno de $100 \mathrm{GPa}$ e manteve-se praticamente constante até a temperatura de $125^{\circ} \mathrm{C}$. Esse valor de temperatura corresponde à temperatura de inicio da transição vítrea. Dessa forma a temperatura de aquecimento máxima para os compósitos CFRP/NiTi obtidos com esse tipo de matriz foi limitada por esse valor de temperatura $\mathrm{T}_{\mathrm{g}}$ do CFRP, por se tratar de um limite em que o material passa por uma grande diminuição de sua rigidez devido a diminuição da viscosidade da resina. Consequentemente, acima dessa temperatura deve ocorrer também diminuição das propriedades de aderência que fazem com que o carregamento seja transferido para as fibras de reforço e fios NiTi. Os resultados em DMA evidenciam que o CFRP apresenta estabilidade térmica até aproximadamente $125^{\circ} \mathrm{C}$, temperatura que é proxima a temperatura final de ativação dos fios NiTi ( $\left.\mathrm{A}_{f}\right)$. Dessa forma, os fios NiTi podem sofrer transformação martensitica reversa por aquecimento sem que a integridade da matriz de compósito CFRP seja comprometida.

Uma análise teórica usando a regra das misturas ${ }^{[7]}$, em que levou-se em consideração que o módulo de elasticidade do compósito CFRP/NiTi seria igual à média ponderada da fração volumétrica e do módulo de cada constituinte (CFRP e NiTi). Essa análise mostrou a necessidade de produção de um compósito com rigidez (módulo elasticidade) próximo ao dos fios NiTi na temperatura ambiente $\left(\sim 30^{\circ} \mathrm{C}\right)$ para a obtenção de um melhor efeito de ativação, correspondente a um aumento de rigidez do compósito CFRP/NiTi quando do seu aquecimento. Tendo em vista que o módulo

Tabela 1. Temperaturas de transformação de fase reversa (martensita - austenita) para os fios NiTi tratados e treinados.

\begin{tabular}{|c|c|c|c|c|c|}
\hline \multirow{2}{*}{ Fios $N i T i$} & \multicolumn{2}{|c|}{$\operatorname{Ai}\left({ }^{\circ} \mathrm{C}\right)$} & \multicolumn{2}{|c|}{$A_{F}\left({ }^{\circ} C\right)$} & \multirow{2}{*}{$\frac{\Delta \mathrm{E}(\mathrm{G} \mathrm{Pa})}{D M A}$} \\
\hline & $D M A$ & $D S C$ & $D M A$ & $D S C$ & \\
\hline Liga M Tratada & 72,1 & 47,2 & 78,3 & 59,2 & 31,6 \\
\hline Liga M Treinada & 74,3 & 55,9 & 106,5 & 61,4 & 27,1 \\
\hline Liga H Tratada & 99,2 & 80,9 & 117,2 & 88,0 & 23,9 \\
\hline Liga H Treinada & 91,0 & 73,0 & 109,3 & 76,4 & 13,4 \\
\hline
\end{tabular}


de elasticidade do CFRP puro alinhado é da ordem de $100 \mathrm{GPa}$ e que o valor dessa propriedade para os fios NiTi a temperatura ambiente é de aproximadamente $25 \mathrm{GPa}$, deve-se aperfeiçoar o sistema de forma a se produzir uma matriz de compósito CFRP com um módulo de elasticidade próximo aos fios NiTi e com uma boa aderência entre as camadas de pré-preg, bem como entre estas camadas e os fios NiTi. Esse fato levou a necessidade de realizar uma investigação do comportamento do compósito CFRP/NiTi com as fibras de carbono em diferentes ângulos $\theta$ em relação aos fios NiTi (Figura 1).

Na Figura 6 apresenta-se o comportamento do módulo de elasticidade dos compósitos CFRP puros, com as fibras de carbono unidirecionais dispostas em diferentes ângulos $\theta\left(45,40,35,30,25,20,15,10,5\right.$ e $\left.0^{\circ}\right)$ em relação ao eixo longitudinal da amostra. Os ensaios em DMA foram realizados no modo de flexão simples (single cantilever). Para esses compósitos todas as camadas de CFRP foram dispostas na mesma angulação.

É possível observar da Figura 6a que o módulo de elasticidade do CFRP sofre uma grande diminuição com o aumento do ângulo das fibras de carbono em relação à direção longitudinal das amostras e que a estabilidade térmica até $125{ }^{\circ} \mathrm{C}$ é mantida independente do ângulo utilizado. Assim sendo, espera-se que o CFRP com as fibras alinhadas em $15^{\circ}$ possa representar uma boa opção para confecção de compósitos CFRP/NiTi. Nesse caso, estima-se que não haverá grandes descontinuidades como vazios entre camadas de fibras, regiões com aglomerados de resina, entre outros, pelo fato das fibras de carbono estarem dispostas a um ângulo relativamente pequeno em relação aos fios NiTi distribuídos longitudinalmente nas amostras. Ao mesmo tempo, esse compósito apresenta um módulo de elasticidade na direção longitudinal ( $37 \mathrm{GPa})$ próximo ao dos fios NiTi antes da sua transformação de fase $(\sim 25 \mathrm{GPa})$ e inferior quando os fios estiverem no estado austenítico, devendo conferir ao compósito ativo CFRP/NiTi um bom grau de funcionalidade e ativação. A temperatura $T_{g}$ não muda com a variação do ângulo das fibras de reforço pois é uma propriedade intrínseca do sistema epóxi usado no CFRP.

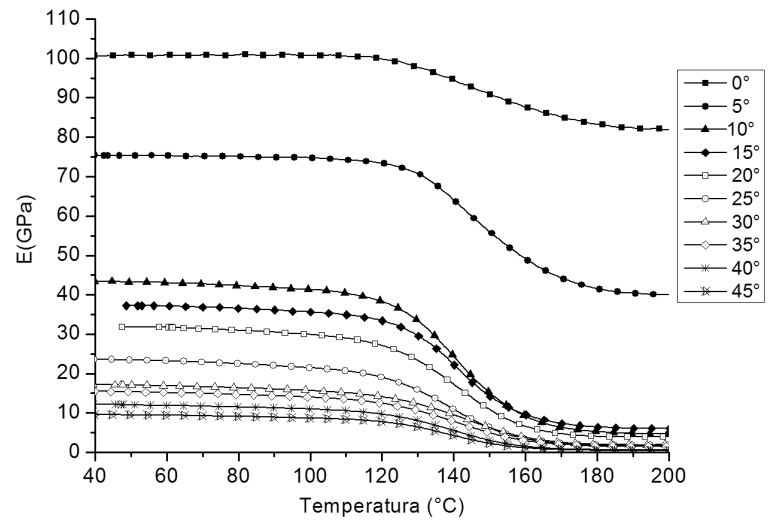

(a)
Apresenta-se na Figura 7 o comportamento do módulo de elasticidade em função da temperatura para um compósito CFRP/NiTi-M com 19,8\% de fração volumétrica de fios NiTi.

O comportamento mostrado na Figura 7 se assemelha qualitativamente a resultados obtidos por outros autores ${ }^{[13,14]}$, com um aumento do módulo de elasticidade na faixa de temperatura em que ocorre a transformação de fase dos fios NiTi. Um comportamento semelhante ocorreu para as demais amostras utilizadas nesse estudo, em que divergiram apenas em relação à variação do ganho de módulo de elasticidade $(\Delta \mathrm{E})$, conforme mostra-se na Figura 8.

Pelos resultados obtidos nos testes realizados em DMA verificou-se que a ativação dos fios NiTi propicia um aumento da rigidez do CFRP/NiTi. O módulo de elasticidade dos compósitos CFRP/NiTi acima da temperatura de transformação de fase reversa dos fios $\operatorname{NiTi}\left(\mathrm{A}_{\mathrm{f}}\right)$ aumentam em relação aos valores observados na temperatura antes da transformação, o que possibilita a utilização dessas estruturas em temperaturas mais elevadas (inferiores a $\mathrm{T}_{\mathrm{o}}$ ), da ordem de $100^{\circ} \mathrm{C}$, sem comprometimento das propriedades termomecânicas do sistema.

Inicialmente, a introdução dos fios NiTi longitudinalmente no CFRP com fibras alinhadas a $15^{\circ}$ fez com que o módulo de elasticidade do compósito CFRP/NiTi $(\sim 29,5 \mathrm{GPa})$ diminuísse em relação aos valores obtidos para a amostras com fibras a $15^{\circ}$ sem fios $(\sim 37,5 \mathrm{GPa})$ e na temperatura ambiente $\left(\sim 30^{\circ} \mathrm{C}\right)$. Esse fato é previsto pela regra das misturas, já que se introduziu um material com propriedades inferiores à da matriz. A grande relevância desse trabalho está na obtenção de um compósito com capacidade de desempenhar controle através da variação do seu módulo de elasticidade com o aquecimento. Durante a ativação do compósito observa-se que suas propriedades se igualam e até mesmo aumentam (dependendo da fração volumétrica de fios NiTi) em relação aos valores observados na temperatura antes da transformação para os compósitos sem fios.

Na Figura 8 apresenta-se os ganhos percentuais de aumento de módulo de elasticidade $(\% \Delta \mathrm{E})$ para os compósitos ativos CFRP/NiTi ensaiados nos dois modos (tração e flexão).

Para todos os casos observou-se que o ganho de módulo de elasticidade $(\Delta \mathrm{E})$ aumenta com o aumento da fração

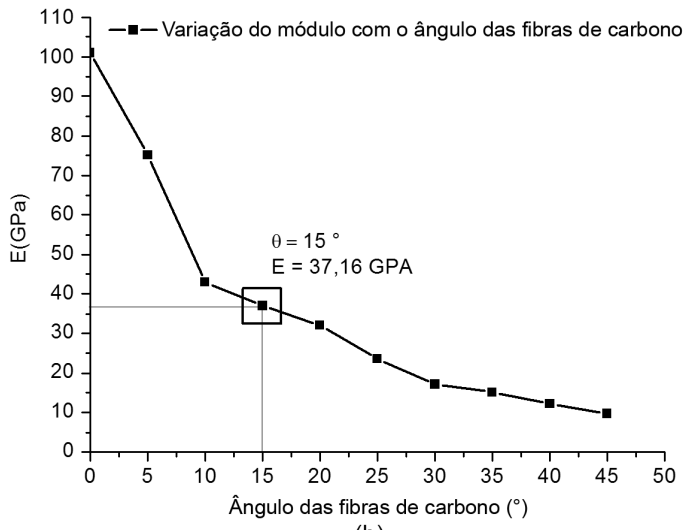

(b)

Figura 6. Variação do módulo de elasticidade em função da temperatura (a) e na temperatura ambiente (b) para compósitos CFRP com diferentes ângulos. 
volumétrica dos fios NiTi até um valor limite em que, mesmo aumentando a fração desses fios, o ganho começa a decrescer. Conforme observado, houve decréscimo da $\% \Delta \mathrm{E}$ para frações volumétricas de fios NiTi em torno de $19 \%$. Este fenômeno deve-se a descontinuidades entre os fios NiTi e o CFRP. O CFRP suporta certa quantidade de fios NiTi sem que sua estrutura seja comprometida. A partir de um dado valor a heterogeneidade fica elevada e a transferência de carga entre a matriz de CFRP a $15^{\circ} \mathrm{e}$ os fios NiTi torna-se fraca, comprometendo a integridade

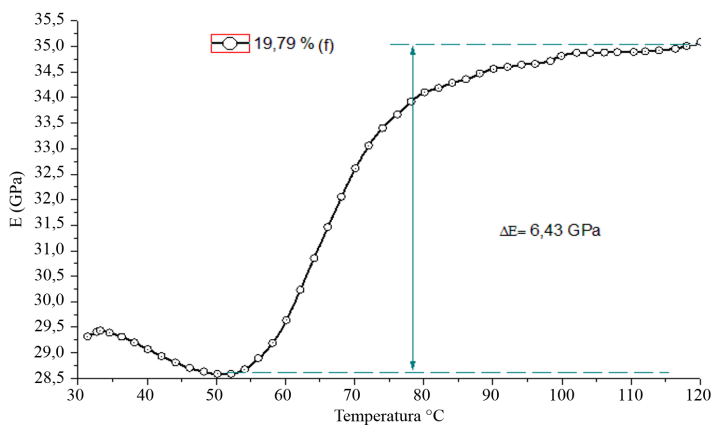

Figura 7. Comportamento típico da variação do módulo de elasticidade em função da temperatura para uma das amostras de CFRP/NiTi-M.

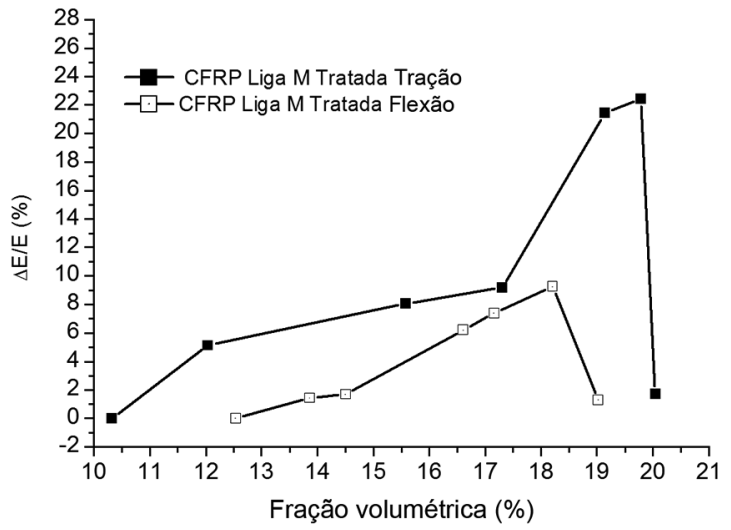

(a)

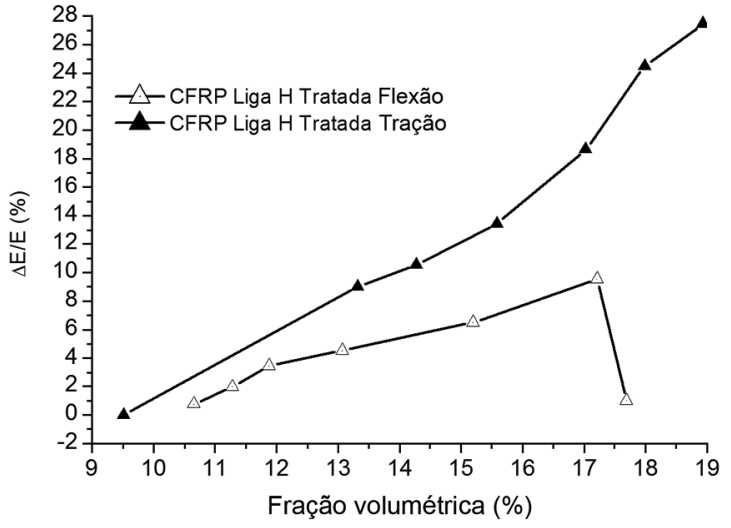

(c) e assim as propriedades do compósito. Com o aumento do número de fios NiTi chega-se a uma situação em que as camadas de CFRP (inferior e superior) quase não se tocam fazendo com que haja uma fraca interface entre os constituintes. Na Figura 9 apresenta-se uma imagem de microscopia ótica de um compósito de CFRP/NiTi para o qual a fração volumétrica de fios NiTi é elevada (superior a $20 \%$ ). Para este caso verifica-se que quase não há contato entre as camadas de CFRP superior e a inferior.

Os compósitos ativos CFRP/NiTi testados no modo de tração ao invés de flexão simples (single cantilever) apresentaram maiores ganhos de rigidez em função da fração volumétrica quando os fios NiTi são ativados, mesmo utilizando frações volumétricas aproximadamente iguais.

Apenas o compósito CFRP/NiTi com fios da liga $\mathrm{H}$ treinados divergiu desse comportamento para frações volumétricas maiores que $16 \%$. Esse fenômeno deve-se provavelmente ao fato de que os fios NiTi estão posicionados ao longo da linha neutra dos compósitos e durante a flexão simples a linha neutra do compósito não é afetada, ou seja, não fica sujeita a esforços ${ }^{[15]}$. Já para o teste em modo de tração, a carga é aplicada no sentido axial aos fios NiTi e os mesmos participam inteiramente, recebendo o esforço mecânico na mesma intensidade da matriz de CFRP.

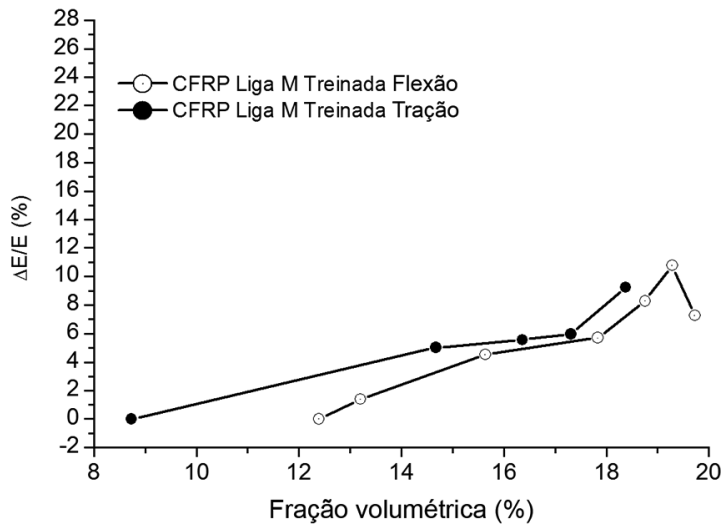

(b)

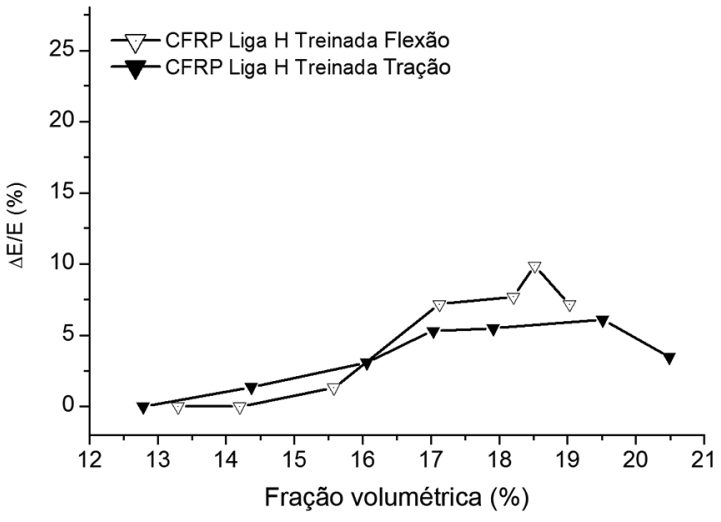

(d)

Figura 8. Comparação do aumento percentual de módulo de elasticidade para os compósitos ativos CFRP/NiTi ensaiados em dois modos (tração - liga M (a), flexão - liga M (b), tração - liga H (c) e flexão - liga H (d)). 


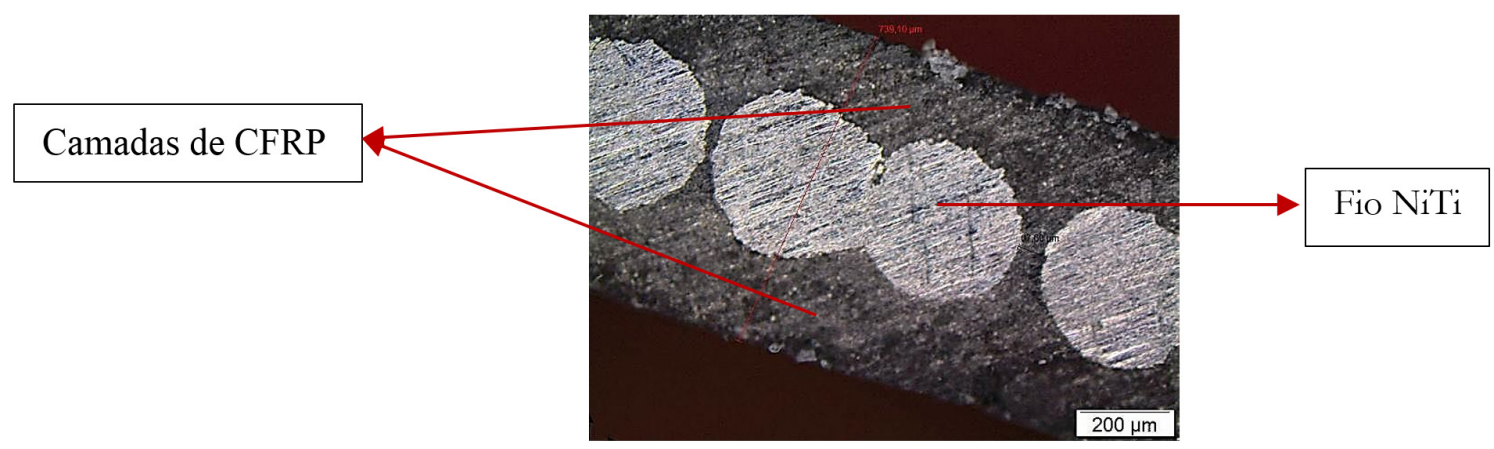

Figura 9. Imagem de microscopia ótica de um compósito CFRP/NiTi com elevada fração volumétrica de fios NiTi (superior a 20\%).

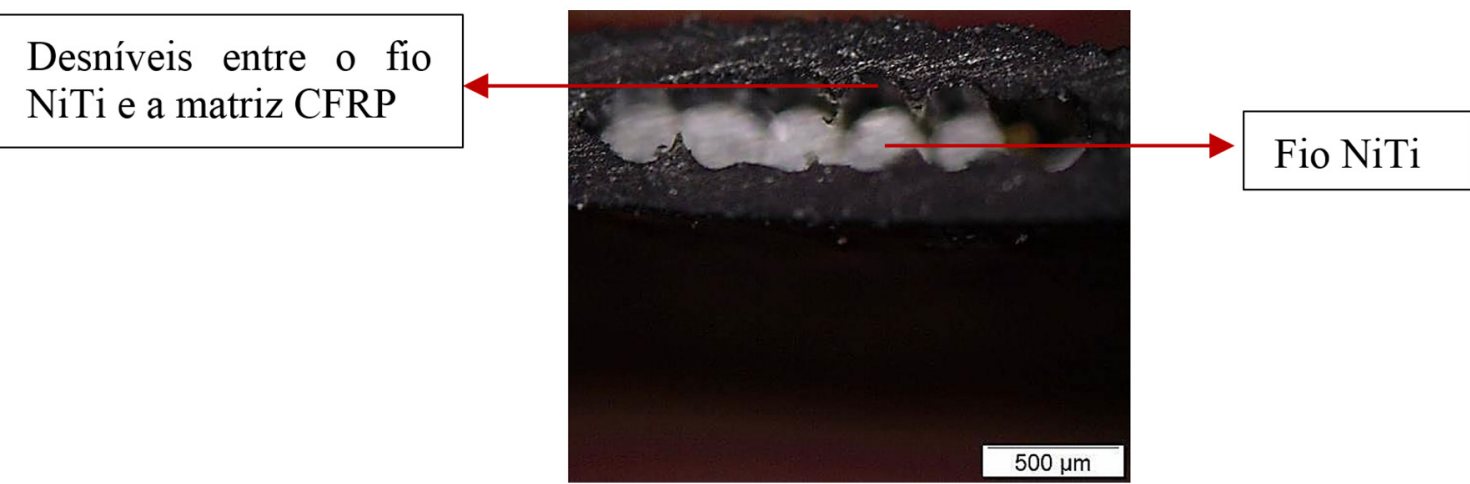

Figura 10. Imagem de microscopia ótica de compósitos CFRP/NiTi mostrando o efeito da auto contração dos fios NiTi treinados na matriz CFRP (aumento de 5x).

Fazendo um comparativo entre os testes realizados em tração e em flexão na Figura 8, observa-se ainda que os compósitos produzidos com os fios NiTi treinados apresentam sempre menores valores de ganho de rigidez em relação aos compósitos com fíos apenas tratados, sendo esse comportamento mais acentuado para os testes realizados em tração. Esse fenômeno está de acordo com a Figura 4 que mostra que o aumento de módulo de elasticidade nos fios NiTi tratados e treinados é menor do que nos fios simplesmente tratados termicamente. Além disso, os fios NiTi treinados, contrariamente aqueles simplesmente tratados, tendem a se contrair por efeito memória de forma quando aquecidos e a interface entre os constituintes pode não ser suficiente para manter a transferência de carga dos fios NiTi para a matriz CFRP levando a uma perda de adesão na interface entre o fio NiTi e a matriz CFRP. Esse comportamento pode ocorrer pelo fato de que a resina quando aquecida fica menos viscosa e assim o fio NiTi treinado, sujeito a contração por efeito memória de forma, pode acabar deslizando dentro da matriz CFRP, danificando as propriedades de fronteira. Na Figura 10 mostra-se uma imagem de microscopia ótica de um compósito CFRP/NiTi com fios NiTi treinados. Nessa imagem se observa, através de desníveis entre o fio NiTi e a matriz CFRP, que após o aquecimento ocorreu contração dos fios dentro da matriz.

Da Figura 8 observa-se também que para menores frações volumétricas de fios NiTi (até 14\%) apenas tratados termicamente obteve-se maiores aumentos de módulo de elasticidade com fios da liga $\mathrm{H}$ do que com os da liga $\mathrm{M}$, sendo esse fenômeno observado para os dois modos de ensaios (flexão e tração). O maior valor de ganho de rigidez $(\sim 28 \%)$ foi observado para o compósito ativo CFRP/NiTi com fios NiTi da liga $\mathrm{H}$ apenas tratada, quando ensaiada no modo de tração uniaxial e com o maior valor de fração volumétrica $(\sim 18,5 \%)$. Para o compósito obtido com fios NiTi da liga M, o melhor resultado de ganho de rigidez $(\sim 23 \%)$ também foi observado com os fios apenas tratados termicamente e com o compósito ativo ensaiado no modo de tração. Conforme já discutido, esse fenômeno está de acordo com a Figura 4 que mostra que o aumento de módulo de elasticidade nos fios NiTi tratados e treinados é menor do que nos fios simplesmente tratados termicamente, devido a introdução de defeitos internos durante o processo de ciclagem dos fios. Além disso, os fios da liga $\mathrm{M}$ não terão sua transformação martensítica completamente atingida, pelo fato da faixa de temperatura empregada nos testes não possibilitar a transformação total para a fase martensita.

\section{Conclusões}

Neste trabalho foi investigada a obtenção de compósitos ativos a partir de pré-impregnados de CFRP aeronáutico e fios funcionais de ligas NiTi com efeito de memória de forma. O potencial de ativação foi avaliado como a capacidade do compósito CFRP/NiTi aumentar de rigidez durante o aquecimento até uma temperatura máxima limitada pela temperatura de transição vítrea da resina epóxi da matriz CFRP. Foi constatado que para obter um aumento de rigidez, medido com base no ganho de módulo de elasticidade do compósito durante o aquecimento, a matriz de CFRP puro 
deve ter um módulo de elasticidade de valor próximo ao dos fios funcionais de NiTi. Portanto, foi necessário utilizar o CFRP com as fibras de carbono formando um ângulo de $15^{\circ}$ com a direção dos fios NiTi. A realização de ensaios de DMA dos compósitos ativos CFRP/NiTi nos modos de tração e flexão demonstrou que compósitos com a mesma fração volumétrica de fios NiTi apresentam um maior potencial de ativação quando solicitados em tração. Além disso, os fios NiTi no estado simplesmente tratado termicamente apresentam-se como melhor alternativa para a obtenção de compósitos ativos do tipo CFPR/NiTi. Isso decorre do maior aumento de módulo de elasticidade com o aumento da temperatura (chega-se a dobrar de valor) associado a uma pequena variação de temperatura (de aproximadamente $15^{\circ} \mathrm{C}$ ), comparativamente aos fios NiTi tratados termicamente e submetidos a um processo de treinamento de estabilização. Verificou-se também que a utilização de fios NiTi da liga H, de temperaturas de transformação mais altas, simplesmente submetidos a tratamento térmico, possibilita a fabricação de compósitos CFRP/NiTi com maior potencial de ativação por estarem completamente no estado martensítico na temperatura ambiente e dessa forma ocorre uma transformação de fase completa quando o compósito ativo é aquecido.

Finalmente, este estudo permitiu constatar que os compósitos ativos obtidos utilizando o CFRP como matriz para incorporação dos fios atuadores NiTi, representam uma excelente opção de material para aplicação em projetos aeronáuticos, apresentando propriedades mecânicas e potencial ativo bem superiores às já observadas em compósitos que utilizam resinas poliméricas puras e de alumínio como matriz, podendo ser aplicados como sensores de dilatação e de temperatura, para controles de rigidez, amortecimento e consequentemente de vibração, entre outras aplicações especificas que podem requerer o potencial ativo que estas estruturas podem fornecer.

\section{Agradecimentos}

Os autores agradecem ao CNPq pelo financiamento dos seguintes projetos: INCT de Estruturas Inteligentes

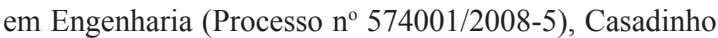
UFCG-UFRJ-ITA (Processo n ${ }^{\circ}$ 552199/2011-7), CT-Aeroespacial 22/2013 (Processo n ${ }^{\circ} 402082 / 2013-3$ ) e PQ 2 (Processo no 302320/2011-3). Um agradecimento especial também a CAPES pelo financiamento da bolsa de doutorado de Z. T. Vilar e ao Engenheiro Edmundo Ortiz, da Hexcel Composites, pela doação dos pré-impregnados de CFRP usados neste trabalho.

\section{Referências}

1. Tebaldi, A., Coelho, L. S., \& Lopes, V., Jr. (2006). Detecção de falhas em estruturas inteligentes usando otimização por nuvem de partículas: fundamentos e estudo de casos. Revista SBA: Controle \& Automação, 17(3), 312-330. http://dx.doi. org/10.1590/S0103-17592006000300006.
2. Michaud, V. (2003). Can shape memory alloy composites be smart? Scripta Materialia, 50(2), 249-253. http://dx.doi. org/10.1016/j.scriptamat.2003.09.016.

3. Jang, B., \& Kishi, T. (2005). Thermomechanical response of TiNi fiber-impregnated CFRP composites. Materials Letters, 59(19-20), 2472-2475. http://dx.doi.org/10.1016/j. matlet.2005.03.027.

4. Tsoi, K. A., Schrooten, J., Zheng, Y., \& Stalmans, R. (2002). Thermal response of glass fibre/epoxy composites with embedded TiNiCu alloy wires. Materials Science and Engineering A, 335(1-2), 157-163. http://dx.doi.org/10.1016/ S0921-5093(01)01912-8.

5. Araújo, C. J., Rodrigues, L. F. A., Coutinho, J. F., No., \& Reis, R. P. B. (2008). Fabrication and static characterization of carbon-fiber-reinforced polymers with embedded NiTi shape memory wire actuators. Smart Materials and Structures, 17(6), 1-8. http://dx.doi.org/10.1088/0964-1726/17/6/065004.

6. Hexcel Composites. (2014). Stamford. Recuperado em 12 de setembro de 2014, de http://www.hexcel.com/Resources/ DataSheets/Prepreg-Data-Sheets/8552_us.pdf

7. Vilar, Z. T. (2013). Compósitos poliméricos reforçados com fibras de carbono (CFRP) incorporando fios atuadores de NiTi com memória de forma: caracterização termomecânica usando análise mecânica dinâmica (DMA) (Tese de doutorado). Universidade Federal de Campina Grande, Campina Grande.

8. Xu, Y., Otsuka, K., Yoshida, H., Nagai, H., Oishi, R., Horikawa, H., \& Kishi, T. (2002). A new method for fabricating SMA/ CFRP smart hybrid composites. Intermetallics, 10(4), 361-369. http://dx.doi.org/10.1016/S0966-9795(02)00011-0.

9. American Society for Testing and Materials-ASTM. (2014). ASTM F2082-06: standard test method for determination of transformation temperature of nickel-titanium shape memory alloys by bend and free recovery. West Conshohocken: ASTM. Recuperado em 12 de setembro de 2014, de http://www.astm. org/Standards/F2082.htm

10. Otsuka, K., \& Wayman, C. M. (1998). Shape memory materials. Cambridge: Cambridge University Press. 284 p.

11. Memory-Metalle. (2014). NiTinol alloy types, conditions and surfaces. Connecticut. Recuperado em 12 de setembro de 2014, de http://www.memory-metalle.de/html/03_knowhow/PDF/ MM_13_alloytypes_e.pdf

12. Vilar, Z. T., Araujo, C. J., Reis, R. P. B., \& Travassos, G. D. (2010). A critical analysis on the use of dynamic-mechanical analyzer (DMA) in the study of shape memory alloys. In Anais do IX Encontro da SBPMat. Ouro Preto.

13. Ni, Q., Zhang, R., Natsuki, T., \& Iwamoto, M. (2007). Stiffness and vibration characteristics of SMA/ER3 composites with shape memory alloy short fibers. Composite Structures, 79(4), 501-507. http://dx.doi.org/10.1016/j.compstruct.2006.02.009.

14. Leal, A. S. C., Araújo, C. J., Silva, S. M. L., \& de Lima, A. G. B. (2013). Development and characterization of active nanocomposites with embedded shape memory alloy wires. Materialwissenschaft und Werkstofftechnik, 44(12), 972-976. $\mathrm{http} / / / \mathrm{dx}$. doi.org/10.1002/mawe.201300132.

15. Hibbeler, R. C. (2010). Resistência dos materiais (7. ed., pp. 181-251). São Paulo: Pearson Prentice Hall.

Enviado: Set. 12, 2014

Revisado: Fev. 28, 2015

Aceito: Mar. 30, 2015 